\title{
Geometric Nonlinear Analysis for Frame Structures, Part 1: Static Analysis
}

\author{
Duan Jin ${ }^{1, a}$, Li Yun-gui ${ }^{1}$ \\ ${ }^{1}$ China State Construction Technical Center, Beijing, 101300, PR China \\ a duanjin78@126.com
}

\begin{abstract}
Key words: structural simulation, system integration, finite element analysis
\end{abstract}
Abstract: In this paper, the governing equation of static analysis for frame structures is presented with the geometric nonlinearity considered. In the process of derivation, the updated Lagrange format is adopted. It is deduced for general three-dimensional solid elements first and then simplified for one-dimensional beam elements. The elemental equilibrium equation is established at current configuration and then transformed into the initial configuration. Finally, a numerical example about the pure bending analysis of a cantilever beam subjected to a concentrated load at the end-point is discussed to demonstrate the validity and accuracy of the present method.

\section{Introduction}

Geometrically nonlinear analysis is a classical problem. Several century ago, it has attracted much attention of some scholars, such as Jacob Bernoulli (1654-1705) and Euler (1707-1783) [1]. However, due to its complicities, most of the engineering problem is analyzed with the geometrical nonlinearity ignored, until the Finite Element Method (FEM) arising in the middle of last century. In 1956, Turner、Clough、Martin \& Topp [2] published a paper using finite element method to analyze complicated structure, i.e. wings of airplane. In 1960, Clough [3] defined formally the terminology of Finite Element Method, FEM for short. Ever from then on, FEM has been widely used in all kinds of engineering. And the problem of geometrical nonlinearity has attracted many scholars and engineers using FEM. Generally speaking, there are two kinds of methods widely used in the geometrically nonlinear analysis, i.e. Total Lagrange method (TL) and Updated Lagrange method (UL). Bathe\& Bolourchi [4] pointed out that UL and TL are essentially equivalent while UL is usually more efficient.

In this paper, the governing equation of static analysis for frame structures is presented with the geometric nonlinearity considered. In the process of derivation, the updated Lagrange format is adopted. It is deduced for general three-dimensional solid elements first and then simplified for one-dimensional beam elements. The elemental equilibrium equation is established at current configuration and then transformed into the initial configuration. Finally, a numerical example about the pure bending analysis of a cantilever beam subjected to a concentrated load at the end-point is discussed to demonstrate the validity and accuracy of the present method.

\section{The governing equation for static analysis considering geometric nonlinearity}

Motion of body in Cartesian co-ordinate system is illustrated in Figure 1, and it is assumed that the configurations are known at time $0, \Delta t, 2 \Delta t, \ldots$, and time $t$, while un-known at time $t+\Delta t$. Selecting the configuration at time $t$ as the reference system, and considering the fact that the body is balanced at time $t+\Delta t$, the following equation can be obtained by the virtual work principle: 


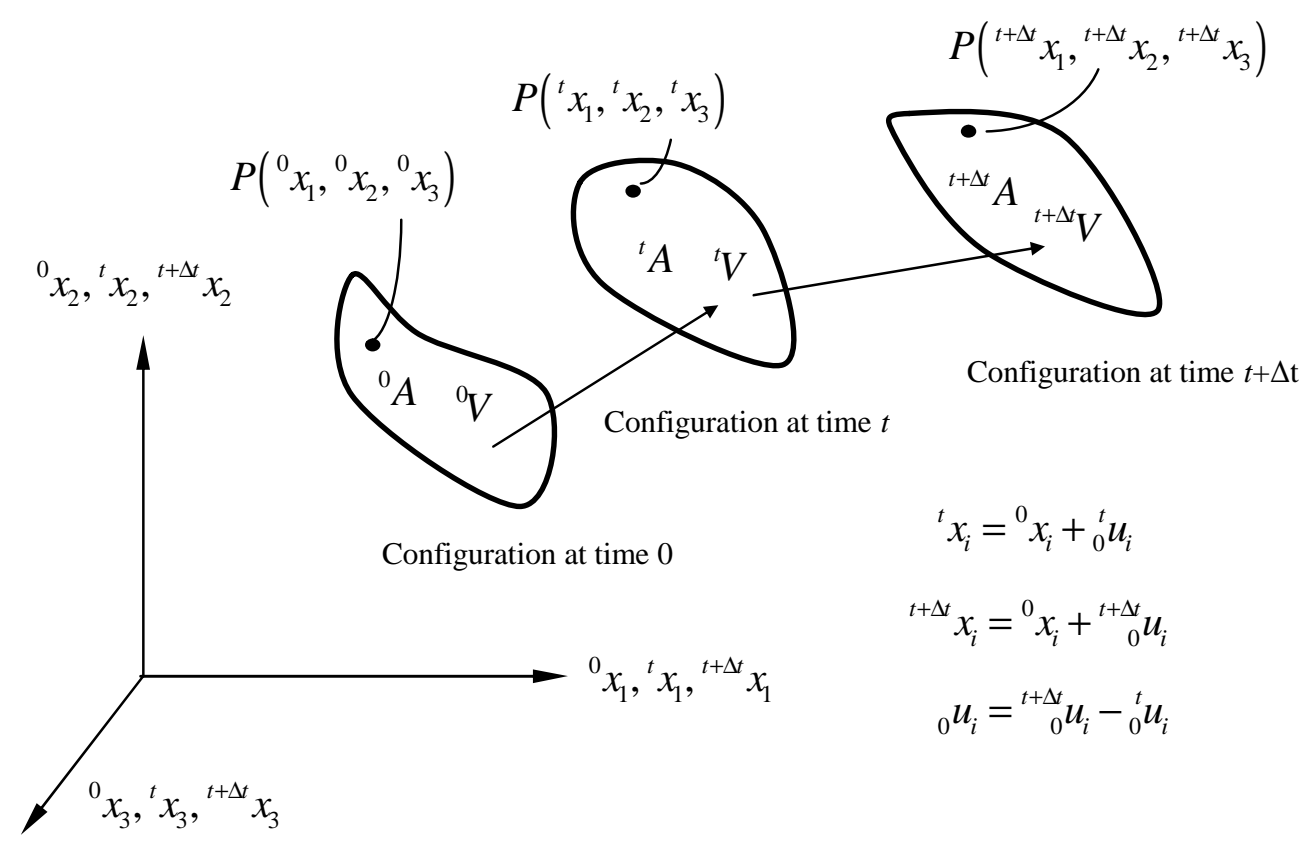

Figure 1 Motion of body in Cartesian co-ordinate system ${ }^{[4]}$

$$
\int_{t_{V}}{ }^{t+\Delta t}{ }_{t} S_{i j} \delta^{t+\Delta t}{ }_{t} \varepsilon_{i j}{ }^{0} d V={ }^{t+\Delta t} \mathrm{~A}
$$

Where ${ }^{t+\Delta t} S_{i j}$ and ${ }^{t+\Delta t} \varepsilon_{i j}$ denotes the second P-K stress tensor and Green strain tensor at time $t+\Delta t$ respectively. They are all use the configuration at time $t$ as the reference system. ${ }^{t+\Delta t} \mathrm{~A}$ denotes the virtual work of external force, e.g. the surface pressure ${ }^{t+\Delta t}{ }_{t} t_{k}$ and body force ${ }^{t+\Delta t} f_{k}$, see the following equation for details.

$$
{ }^{t+\Delta t} \mathrm{~A}=\int_{{ }^{t} A}{ }^{t+\Delta t}{ }_{t} t_{k} \delta{ }^{t+\Delta t}{ }_{t} u_{k}{ }^{t} d A+\int_{{ }^{t} V}{ }^{t} \rho{ }^{t+\Delta t}{ }_{t} f_{k} \delta^{t+\Delta t}{ }_{t} u_{k}{ }^{t} d V
$$

Where ${ }^{t} \rho$ denotes the density at time $t$. if ignoring the change of density along the change of configuration, density is a constant, i.e. ${ }^{t} \rho \equiv{ }^{0} \rho \equiv \rho .{ }^{t+\Delta t} u_{k}$ denotes the displacement at time $t+\Delta t$, and it is measured at the configuration at time $t$

The second P-K stress tensor ${ }^{t+\Delta t} S_{i j}$ can be described in the increment form as following:

$$
{ }^{t+\Delta t} S_{i j}={ }^{t} \tau_{i j}+{ }_{t} S_{i j}
$$

Where ${ }^{t} \tau_{i j}$ denotes the Cauchy stress tensor at time $t,{ }_{t} S_{i j}$ denotes the second P-K stress tensor at time $t$

The relationship of the strain increment from time $t$ to $t+\Delta t$ as ${ }_{t} \varepsilon_{i j}$ and the Green stain tensor at time $t+\Delta t$ can be expressed as the following one

$$
{ }_{t}^{t+\Delta t} \varepsilon_{i j}={ }_{t} \varepsilon_{i j}
$$

Where

$$
{ }_{t} \varepsilon_{i j}={ }_{t} e_{i j}+{ }_{t} \eta_{i j}
$$

Where ${ }_{t} e_{i j}$ and ${ }_{t} \eta_{i j}$ denotes the linear and nonlinear part of the strain increment respectively, see the following equation for details 


$$
{ }_{t} e_{i j}=\frac{1}{2}\left({ }_{t} u_{i, j}+{ }_{t} u_{j, i}\right), \quad{ }_{t} \eta_{i j}=\frac{1}{2}{ }_{t} u_{k, i t} u_{k, j}
$$

The relationship of the second P-K stress increment and the Green strain increment can be expressed as the following equation

$$
{ }_{t} S_{i j}={ }_{t} C_{i j r s t} \varepsilon_{r s}
$$

Where ${ }_{t} C_{i j r s}$ denotes the constitutive tensor

Substituting Equation (3-7) into Equation (1), the general UL format can be obtained as follows

$$
\int_{t_{V}}{ }_{t} C_{i j r s t} e_{r s} \delta_{t} e_{i j}{ }^{t} d V+\int_{t_{V}}{ }^{t} \tau_{i j} \delta{ }_{t} \eta_{i j}{ }^{t} d V={ }^{t+\Delta t} \mathrm{~A} \cdot \int_{t_{V}}{ }^{t} \tau_{i j} \delta_{t} e_{i j}{ }^{t} d V
$$

In this paper, only frame structure is concerned. In other words, only the beam element is concerned. For beam elements, the above equation can be transformed to the following one

$$
\begin{aligned}
& \int_{l} \int_{A}\left[E_{t} e_{x x} \delta_{t} e_{x x}+G_{t} e_{x y} \delta_{t} e_{x y}+G_{t} e_{x z} \delta_{t} e_{x z}+{ }^{t} \tau_{x x} \delta_{t} \eta_{x x}+{ }^{t} \tau_{x y} \delta_{t} \eta_{x y}+{ }^{t} \tau_{x z} \delta_{t} \eta_{x z}\right] d y d z d x \\
& ={ }^{t+\Delta t} \mathrm{~A}-\iint_{l} \iint_{A}\left[{ }^{t} \tau_{x x} \delta_{t} e_{x x}+{ }^{t} \tau_{x y} \delta_{t} e_{x y}+{ }^{t} \tau_{x z} \delta_{t} e_{x z}\right] d y d z d x
\end{aligned}
$$

or in matrix form as follows

$$
\left({ }_{t}^{t} \mathbf{K}_{L}+{ }_{t}^{t} \mathbf{K}_{G}\right){ }_{t} \mathbf{a}^{e^{*}}={ }^{t+\Delta t}{ }_{t} \mathbf{P}_{e}-{ }_{t}^{t} \mathbf{P}
$$

Where ${ }_{t}^{t} \mathbf{K}_{L}$ and ${ }_{t}^{t} \mathbf{K}_{G}$ denotes the linear stiffness matrix and geometrically nonlinear stiffness matrix respectively, ${ }_{t}^{t} \mathbf{P}$ denotes the internal force of element, ${ }^{t+\Delta t}{ }_{t} \mathbf{P}_{e}$ denotes the external load

\section{Numerical example}

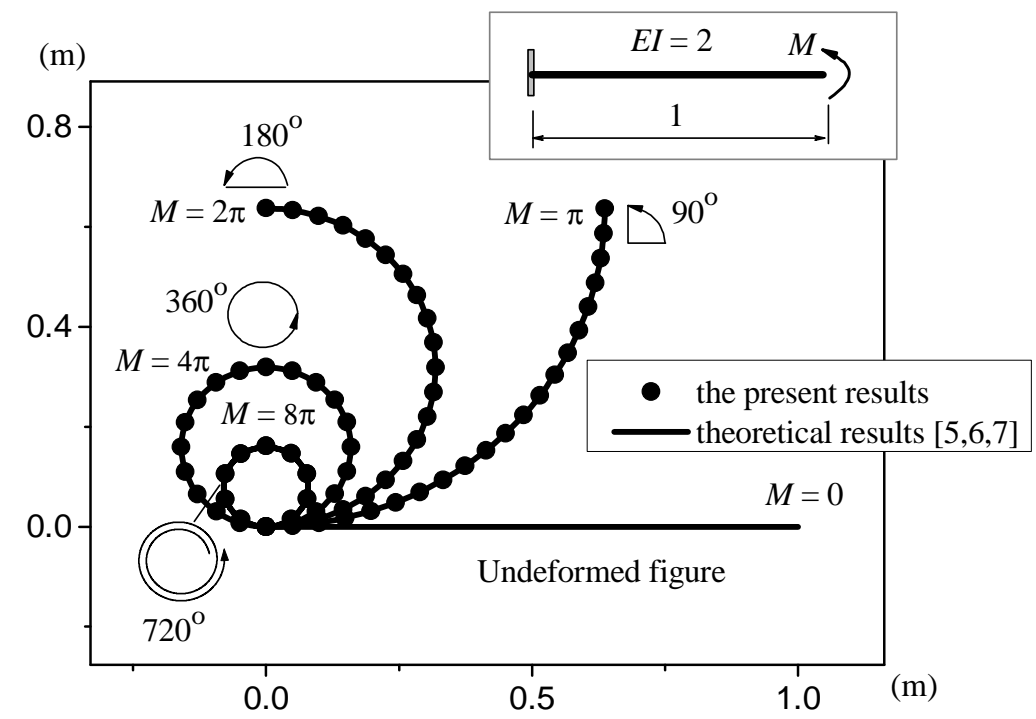

Fig.2 Deformed figures of pure bending beam under different moments

In order to verify the validity of the above method, a numerical example is presented and discussed here. Figure 2 shows a straight rod of unit length and bending stiffness $E I=2\left(\mathrm{~N} . \mathrm{m}^{2}\right)$ subjected to a concentrated end moment $\mathrm{M}$. The theoretical solution to this problem $[5,6,7]$ is a 
circular curve with radius $=E I / M$. Detailedly, an applied moment, $M=\pi$, will force the rod to deform into a quadrant, and a moment twice this magnitude, i.e., $M=2 \pi$, will force the rod to deform into a semicircle, and a moment quadruple this magnitude, i.e., $M=4 \pi$, will force the rod to deform into a full closed circle, and a moment octuple this magnitude, i.e., $M=8 \pi$, will make the rod wind around itself twice. The final shapes of the rod calculated by the present method and theoretical solution are depicted in Fig. 4, in which Good agreement is observed. Furthermore, it could be concluded that the structural stiffness of pure bending beam would not change along with the moment. In other words, the pure bending beam would deform along with the change of end moment, while its stiffness would remain unchanged.

\section{Summary}

In this paper, the governing equation of static analysis for frame structures is presented with the geometric nonlinearity considered. In the process of derivation, the updated Lagrange format is adopted. It is deduced for general three-dimensional solid elements first and then simplified for one-dimensional beam elements. The elemental equilibrium equation is established at current configuration and then transformed into the initial configuration. Finally, a numerical example about the pure bending analysis of a cantilever beam subjected to a concentrated load at the end-point is discussed to demonstrate the validity and accuracy of the present method.

\section{Reference}

[1] Clough R W. Early History of the Finite Element Method from the View Point of a Pioneer. International Journal of Numerical Methods in Engineering, 2004, 60: 283-287

[2] Turner M J, Clough R W, Martin H C, Topp L J. Stiffness and Deflection Analysis of Complex Structures. Journals of Aerospace Science, 1956, 23:805-823

[3] Clough R W. The Finite Element Method in Plane Stress Analysis. Proceedings of the Second ASCE Conference on Electronic Computation, Pittsburgh, PA, 1960

[4] Bathe K J, Bolourchi S. Large Displacement Analysis of Three-Dimensional Beam Structures. Int. J. Num. Mech. Engng., 1979, 14: 961-986

[5] M. Schulz and F.C. Filippou, Non-linear Spatial Timoshenko Beam Element with Curvature Interpolation, International Journal for Numerical Methods in Engineering, vol. 50, pp. 761-785, 2001.

[6] J.C. Simo, a Three Dimensional Finite Strain Rod Model. Part 2: Computational Aspects, Computer Methods in Applied Mechanics and Engineering, vol. 58, pp. 79-116, 1986.

[7] E. Reissner, On a One Dimensional Finite Strain Beam Theory: the Plane Problem, J. Appl. Math. Phys, vol. 23, pp. 795-804, 1972. 